Mappemonde

Revue trimestrielle sur l'image géographique et les formes du territoire

$129 \mid 2020$

Varia

\title{
Représenter et analyser les positions relatives des villes sur 2000 ans
}

Julie Gravier

\section{OpenEdition}

\section{Journals}

Édition électronique

URL : http://journals.openedition.org/mappemonde/4562

DOI : $10.4000 /$ mappemonde.4562

ISSN : 1769-7298

\section{Éditeur}

UMR ESPACE

\section{Référence électronique}

Julie Gravier, "Représenter et analyser les positions relatives des villes sur 2000 ans », Mappemonde [En ligne], 129 | 2020, mis en ligne le 10 novembre 2020, consulté le 26 novembre 2020. URL : http:// journals.openedition.org/mappemonde/4562 ; DOI : https://doi.org/10.4000/mappemonde.4562

Ce document a été généré automatiquement le 26 novembre 2020.

\section{(c) (†) (2)}

La revue Mappemonde est mise à disposition selon les termes de la Licence Creative Commons Attribution - Pas d'Utilisation Commerciale - Partage dans les Mêmes Conditions 4.0 International. 


\title{
Représenter et analyser les positions relatives des villes sur 2000 ans
}

\author{
Julie Gravier
}

Référence de la thèse

GRAVIER J. (2018). Deux mille ans d'une ville en système : proposition d'une démarche appliquée au cas de Noyon. Géographie. Université Panthéon-Sorbonne - Paris I.

1 Partant de l'idée que la ville est un espace produit et que la production de cet espace dépend de mécanismes associés à différents niveaux de l'organisation des sociétés, l'objectif de la thèse est de questionner les processus sous-jacents à l'origine des changements dans la ville. Une partie de ces processus résulte des relations entretenues entre les villes et de leurs positions relatives les unes par rapport aux autres (Pumain, 1997). L'objet de cette recherche porte ainsi sur ce qui unit l'histoire d'une ville avec l'histoire des villes avec lesquelles elle fait système. Elle se fonde sur le cas de Noyon, localisée dans les Hauts-de-France et dont l'origine remonte au I ${ }^{\text {er }}$ siècle apr. J.-C.

2 Pour appréhender la position relative de Noyon par rapport aux autres villes avec lesquelles elle est en interaction, nous avons focalisé notre regard sur trois dimensions : les statuts des villes, leurs inscriptions spatiales dans des territoires et des réseaux de communication et leurs tailles.

\section{Reconstituer les populations des villes dans le temps}

3 Les tailles des villes sont très souvent évaluées grâce à la population. Toutefois, il n'existe pas de données qui soient complètes et harmonisées sur l'ensemble du peuplement urbain français avant le début du XIX siècle. En revanche, les surfaces des espaces urbanisés sont reconstituables pour les périodes anciennes et peuvent être 
envisagées comme entretenant une relation avec leurs populations (Garmy, 2009; Cesaretti et al., 2016). Nous avons ainsi construit dans la thèse une démarche permettant d'estimer les populations des villes pour les périodes préindustrielles à partir de leurs surfaces, en questionnant le rapport entre surface et population. Sachant que les formes d'habiter, et donc les densités des villes, changent entre le $\mathrm{I}^{\mathrm{er}}$ et le XVIII ${ }^{e}$ siècle, cette estimation reste bien entendu une approximation. Le protocole permet toutefois d'obtenir des populations de manière harmonisée et paramétrable pour toutes les villes et pour toutes les périodes étudiées.

Figure 1. Population des villes en système avec Noyon, au moins à un moment, sur 2000 ans

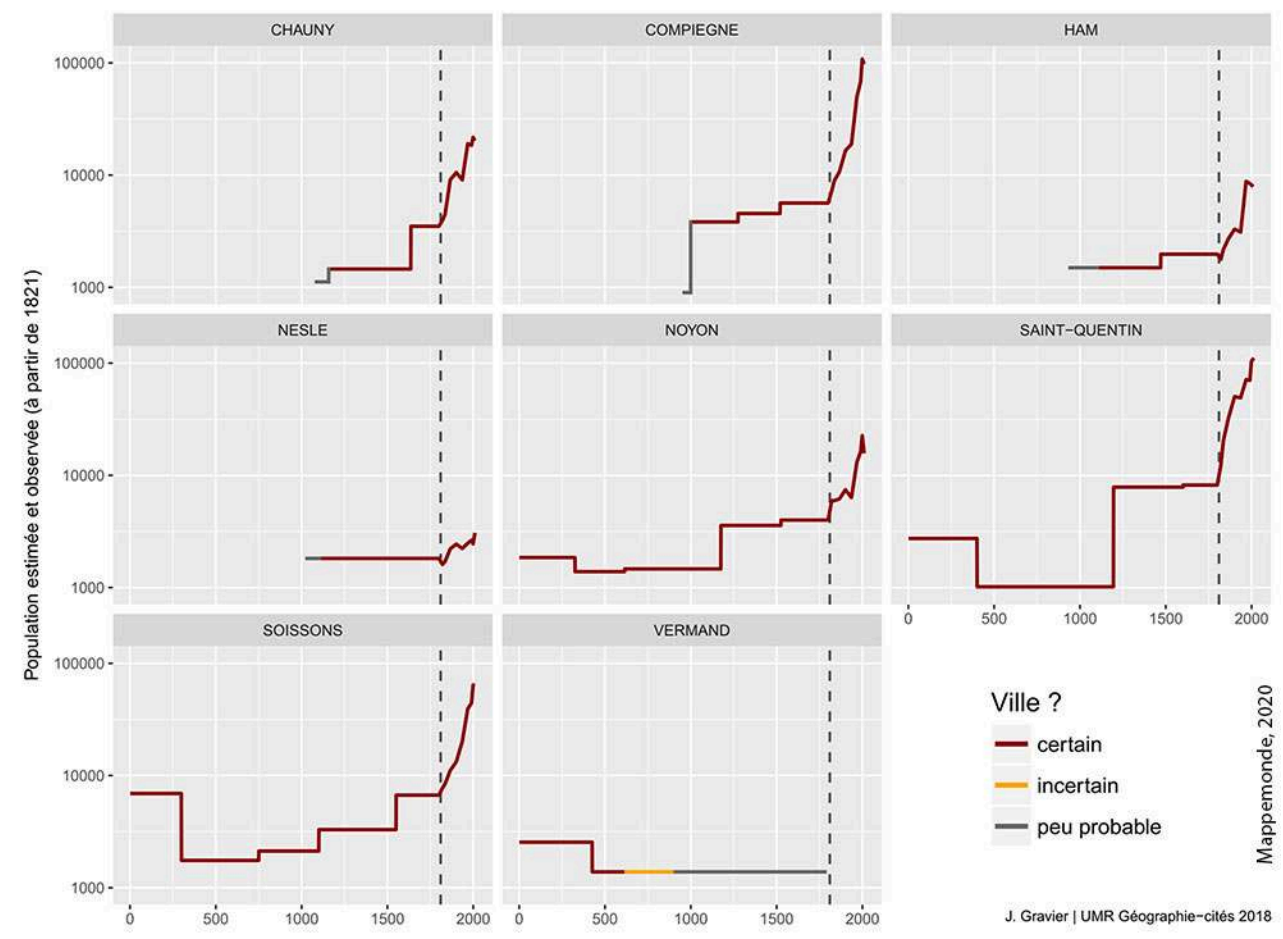

4 Les populations des villes ont ainsi pu être reconstituées ${ }^{1}$ sur toute la durée de leurs existences respectives en tant que "ville» (figure 1). Cette reconstitution permet d'étudier des moments de croissance/décroissance des villes au cours du temps, ainsi que des ressemblances/différences d'intensité de ces phénomènes entre les villes. Il est cependant difficile d'aller plus loin dans l'analyse à partir de ce seul graphique, notamment car il est compliqué d'observer les positions démographiques relatives des villes les unes par rapport aux autres.

\section{Visualiser les trajectoires des positions relatives des villes en système avec Noyon}

5 Nous avons ainsi construit une autre représentation (figure 2). Ce type de graphique dit en coordonnées polaires et figurant habituellement des cycles temporels (jour, semaine, mois) - est ici utilisé pour représenter une double information : d'une part, des pas de temps centennaux (en 1, 101, etc.), et, d'autre part, des classes de taille de ville (petite, moyenne et grande). À chaque pas de temps, les populations reconstituées des villes sont discrétisées en trois classes d'effectifs égaux. 
Figure 2. Trajectoires des positions démographiques relatives des villes en système avec Noyon
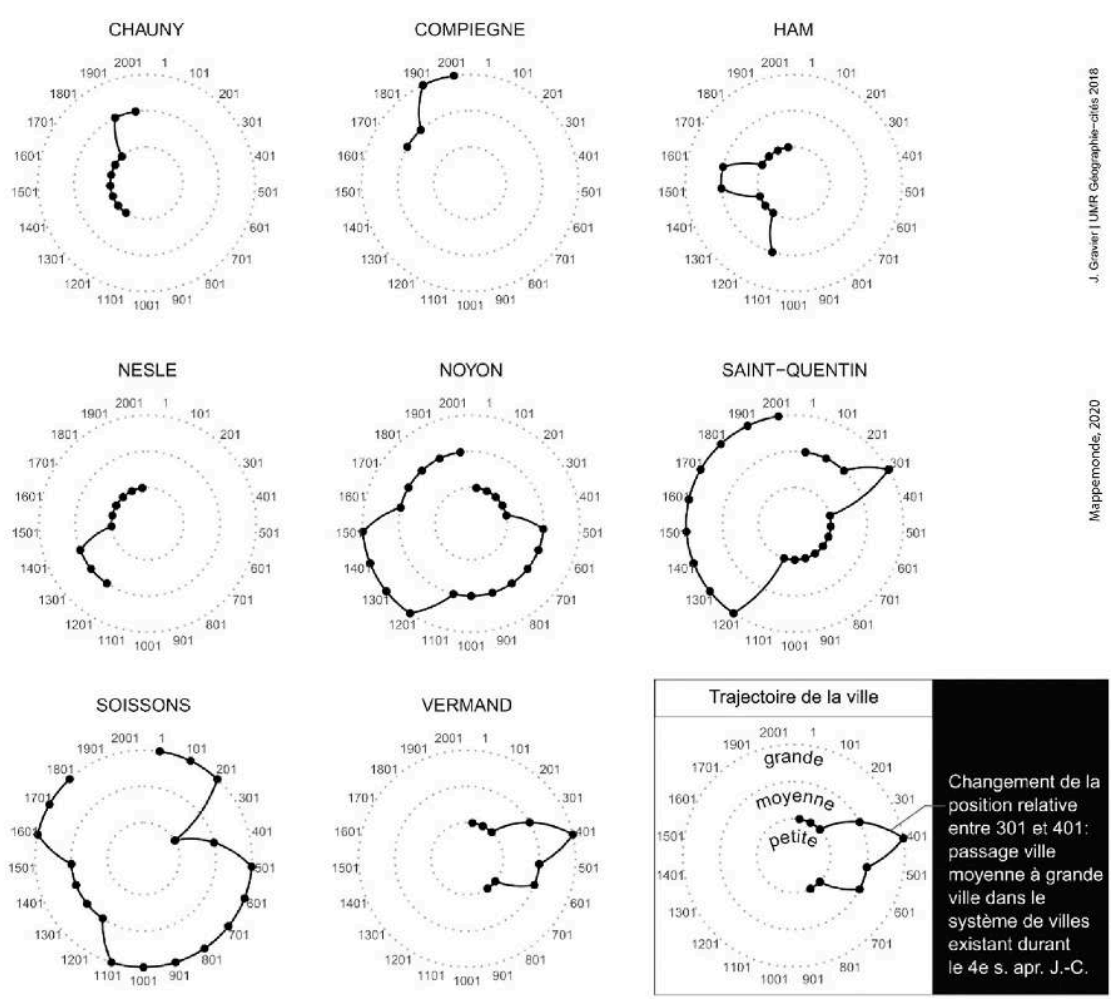
système existant à chaque moment dans le temps. Cela permet de rendre comparables les classes de taille de villes sur 2000 ans et d'intégrer l'évolution du système (apparition/disparition des villes en système avec Noyon); différente permet d'observer rapidement les classes d'appartenance des villes. À titre d'exemple, on remarque directement que la ville de Ham n'a jamais été une grande ville au fil du temps.

fait de la représentation en coordonnées polaires, les longueurs des traits sont plus importantes quand il y a un changement de classe d'une ville. Or, c'est bien l'un des objectifs du graphique que de visualiser d'abord le changement plutôt que la pérennité des situations, et ce, dans le but de mettre en relation ces trajectoires avec les changements de statuts institutionnels de ces mêmes villes au cours du temps.

\section{Soissons et Vermand : deux chefs-lieux de cité romaine en concurrence sur les routes de Reims à Amiens}

10 L'intérêt de ces représentations peut être illustré par le cas de Soissons et Vermand durant la deuxième moitié du IV siècle - début du V siècle. On observe que cette dernière est en décroissance vers le début du $\mathrm{V}^{\mathrm{e}}$ siècle (figure 1), impliquant l'inversion des rangs de Vermand et de Soissons. Soissons est ainsi une grande ville vers 500 tandis 
que Vermand est une ville moyenne (figure 2). Par ailleurs, Noyon, qui est de taille équivalente à Vermand, passe de petite à moyenne ville. Partant du constat de la décroissance de Vermand, nous nous sommes demandé quels mécanismes liés à sa position dans le système de villes auraient pu en être à l'origine.

Notons que les cités des Viromanduens et des Suessions sont incluses dans la province de Gaule Belgique. Son chef-lieu, Reims, était une des étapes de l'axe commercial reliant la capitale des Gaules (Lyon) à Boulogne-sur-Mer - qui traversait notamment les villes d'Autun, Troyes, Reims et Amiens d'après l'itinéraire d'Antonin (Fortia d'Urban et al., 1845). Par ailleurs, il apparaît que deux routes principales permettent de relier Reims à Amiens: l'une, passant par Soissons et Noyon est celle évoquée dans l'itinéraire, l'autre traverse les villes de Saint-Quentin et Vermand (Talbert, 2000 ; McCormick et al. 2013). Les mesures de distance entre Reims et Amiens sur les deux routes permettent d'observer qu'elles sont toutes deux de l'ordre de $150 \mathrm{~km}$ (tableau 1 ) .

Tableau 1. Distance des parcours Reims-Amiens en passant par Soissons ou Vermand durant l'Antiquité romaine

\begin{tabular}{|l|l|l|l|l|l|}
\hline Route 1 & Dist. km & Dist. temps & Route 2 & Dist. km & Dist. temps \\
\hline Reims-Soissons & 54 & $3 \mathrm{~h} 45$ & Reims-St-Quentin & 83 & $5 \mathrm{~h} 45$ \\
\hline Soissons-Noyon & 32 & $2 \mathrm{~h} 15$ & St-Quentin-Vermand & 11 & $45 \mathrm{~min}$ \\
\hline Noyon-Amiens & 61 & $4 \mathrm{~h} 15$ & Vermand-Amiens & 63 & $4 \mathrm{~h} 20$ \\
\hline Total & $\mathbf{1 4 7}$ & - & Total & $\mathbf{1 5 7}$ & - \\
\hline
\end{tabular}

Lecture « Dist. -temps » : considérant qu'un cheval au trot peut parcourir en moyenne environ 14,4 km/ $\mathrm{h}$, dans le cas où l'on doit parcourir $54 \mathrm{~km}$, il faudrait $3 \mathrm{~h} 45$.

12 L'accessibilité entre Reims et Amiens par l'une ou l'autre route est donc équivalente. Cependant, les situations des villes sur ces routes sont différentes. En effet, la répartition spatiale des villes est plus régulière entre Reims et Noyon, où Soissons se trouve presque à mi-chemin, qu'entre Reims et Vermand, la ville de Saint-Quentin se trouvant très proche de cette dernière et très éloignée de Reims. Cette répartition implique que les distances-temps de parcours sont différenciées. On remarque en particulier que le temps de déplacement entre Reims et Vermand est important, sachant en outre, que la distance-temps est ici calculée en prenant en considération un cheval au trot. Or, un cheval ne peut pas trotter en continu sur des distances aussi longues et l'on doit ainsi alterner le pas et le trot ou changer de monture - allongeant le temps de parcours - et cela, sans tenir compte de l'attelage dans le cas de déplacements à caractère commerciaux par exemple. Il apparaît ainsi que les déplacements Reims-Vermand devaient prendre a minima une journée complète, et il est probable que la route passant par Soissons et Noyon ait été privilégiée dans le cas des déplacements commerciaux.

13 Somme toute, la décroissance de Vermand relève probablement de deux mécanismes imbriqués : sa faible centralité dans le réseau routier régional couplée au fait que SaintQuentin, au début $d u V^{e}$ siècle, n'est plus qu'une petite ville du système (figure 2), 
polarisant probablement moins les flux commerciaux sur l'axe Reims-Amiens qu'auparavant.

\section{BIBLIOGRAPHIE}

CESARETTI R., LOBO J., BETTENCOURT L. M. A., ORTMAN S. G., SMITH M. E. (2016). “Population-Area

Relationship for Medieval European Cities". PLoS One. En ligne : http://journals.plos.org/plosone/ article?id=10.1371/journal.pone.0162678

FORTIA D’URBAN A.-J.-F.-X.-P.-E.-S.-P.-A., LAPIE P., MILLER E. (1845). Recueil des itinéraires anciens : comprenant l'Itinéraire d'Antonin, la Table de Peutinger et un choix de périples grecs/avec dix cartes dressées par M. le colonel Lapie. Paris : Imprimerie royale. En ligne : http://gallica.bnf.fr/ark:/12148/bpt6k1030814

GARMY P. (2009). Villes, réseaux et systèmes de villes : contribution à l'archéologie. Habilitation à diriger des recherches, Université Paris I - Panthéon-Sorbonne. En ligne : http://tel.archivesouvertes.fr/tel-00459332/

MCCORMICK M., HUANG G., ZAMBOTTI G., LAVASH J. (2013). “Roman Road Network (version 2008)”.

DARMC Scholarly Data Series, Data Contribution Series \#2013-5, Cambridge : Harvard University, En ligne : http://dataverse.harvard.edu/dataset.xhtml?persistentId=hdl:1902.1/22610

PUMAIN D. « Pour une théorie évolutive des villes ». L'Espace géographique, t. 26, n 2, p. 119-134. En ligne : http://www.persee.fr/doc/spgeo_0046-2497_1997_num_26_2_1063

TALBERT R. J. A. dir. (2000). Barrington Atlas of the Greek and Roman World. Oxford : Princeton University Press.

\section{NOTES}

1. Avant 1821, la reconstitution des populations est effectuée sous forme d'états dans le temps puisque la documentation archéologique et textuelle permet d'identifier les surfaces des villes sur des durées. Après 1821, les recensements des populations sont connus à des dates précises et des inférences linéaires entre ces différentes dates sont réalisées. 